\title{
TITLE:
}

\section{INVERTEBRATE FAUNA OF THE INTERTIDAL ZONE OF THE TOKARA ISLANDS -II. RECORD OF THE STRANDED FLOATING ANIMALS-}

\author{
$\operatorname{AUTHOR}(\mathrm{S}):$ \\ Tokioka, Takasi
}

\section{CITATION:}

Tokioka, Takasi. INVERTEBRATE FAUNA OF THE INTERTIDAL ZONE OF THE TOKARA ISLANDS -II. RECORD OF THE STRANDED FLOATING ANIMALS-. PUBLICATIONS OF THE SETO MARINE BIOLOGICAL LABORATORY 1953, 3(2): 139-139

ISSUE DATE:

1953-12-20

URL:

http://hdl.handle.net/2433/174477

RIGHT: 


\title{
INVERTEBRATE FAUNA OF THE INTERTIDAL ZONE OF THE TOKARA ISLANDS \\ II. RECORD OF THE STRANDED FLOATING ANIMALS1) 2)
}

\author{
TAKASI TOKIOKA
}

Seto Marine Biological Laboratory, Sirahama

The following is the list of the floating animals stranded along the coasts of Takarazima and Nakanosima during my stay.

\section{COELENTERAta}

1. Velella lata Chamisso \& EYsenhardt (Takarazima \& Nakanosima)

2. Porpita umbella O.F. MülLER (Takarazima \& Nakanosima)

3. Physalia physalis utriculus LA MARTINIERE (Takarazima \& Nakanosima)

$$
\text { Mollusca }
$$

4. Janthina janthina (LinNÉ) (Takarazima)

5. Janthina belteata REEVE (Takarazima \& Nakanosima)

6. Janthina (Violetta) globosa SwaINson (Takarazima \& Nakanosima)

7. Janthina (Iodina) umbilicata D'ORBIGNY (Takarazima \& Nakanosima)

8. Recluzia montrouzieri BRAzIER

9. Diacria trispinosa (Lesueur) (Takarazima, 13 shells)

10. Cavolinia uncinata (RANG) (Takarazima, 2 shells)

11. Cavolinia globulosa (RANG) (Takarazima, 2 shells)

12. Nautilus pompilus LINNÉ (Takarazima, pieces)

13. Argonauta hians Solander (Takarazima, one shell)

\section{Crustacea}

14. Lepas pectinata SPENGLER (Takarazima)

15. Lepas anserifera LinNÉ (Takarazima \& Nakanosima)

\section{PROCHORDATA}

16. Thetys vagina (Tilesius) (Takarazima \& Nakanosima)

1) Scientific Survey of the Tokara Islands, Report No. 3.

2) Contributions from the Seto Marine Biological Laboratory, No. 209.

Publ. Seto Mar. Biol. Lab., III (2), 1953. (Article 10) 